\title{
Up-regulation of Fas expression by Pseudomonas aeruginosa- infected endothelial cells depends on modulation of iNOS and enhanced production of NO induced by bacterial type III secreted proteins
}

\author{
MARIA-CRISTINA ASSIS ${ }^{1}$, CARLA FREITAS ${ }^{1}$, ALESSANDRA M. SALIBA ${ }^{1}$, ANA PAULA D'A. CARVALHO ${ }^{1}$, \\ TATIANA A. SIMAO ${ }^{2}$, RODOLPHO M. ALBANO ${ }^{2}$ and MARIA-CRISTINA PLOTKOWSKI ${ }^{1}$
}

Departments of ${ }^{1}$ Microbiology and Immunology, and ${ }^{2}$ Biochemistry, Universidade do Estado do Rio de Janeiro, 20 551-030 Rio de Janeiro, Brazil

Received November 25, 2005; Accepted January 30, 2006

\begin{abstract}
To obtain a better understanding of the mechanisms involved in the up-regulation of the Fas apoptotic signaling cascade induced by $P$. aeruginosa type III secretion system (TTSS), human umbilical vein endothelial cells (HUVEC) were infected with $P$. aeruginosa $\mathrm{PAO}-1$ or its TTSS-negative mutant PAO-1::exsA. PAO-1 was significantly more cytotoxic than the mutant and features of apoptosis (DNA fragmentation and annexin $\mathrm{V}$ reactivity) were more prominent in cultures infected with the wild-type bacteria. PAO-1 induced the upregulation of Fas and the release of soluble FasL (sFasL) from infected cells but cell treatment with antagonist anti-Fas did not completely abrogate apoptosis suggesting that, besides the activated Fas-FasL pathway, other mechanisms are likely to be associated with the induction of apoptosis. LNMMA, a potent inhibitor of NO synthesis, completely inhibited apoptosis in both PAO-1 and PAO-1::exsA infected cultures. Moreover, PAO-1 was shown to up-regulate both the expression of iNOS and NO production by HUVEC. Treatment of cells with LNMMA completely inhibited cell expression of mFas. Based on these results we speculate that $P$. aeruginosa TTSS not only accounts for HUVEC higher expression of Fas and release of sFasL but also leads to overproduction of $\mathrm{NO}$ and to a NO-dependent up-regulation of the Fas-FasL proteins.
\end{abstract}

\section{Introduction}

Apoptosis is an essential mechanism for the homeostasis of the human defense system. However, several lines of evidence

Correspondence to: Dr Maria-Cristina Plotkowski, Department of Microbiology and Immunology - FCM/UERJ, Av. 28 de Setembro, 87 fundos, $3^{\circ}$ andar, 20 551-030 Rio de Janeiro, Brazil

E-mail: crisplot@yahoo.com.br

Key words: P. aeruginosa, apoptosis, type III secretion system, human umbilical vein endothelial cells, NO, iNOS, Fas-FasL system indicate that some bacteria can modulate the apoptotic signaling cascade of host cells as an additional mechanism of pathogenesis (1). Although apoptosis of infected cells limits the replication of invading microorganisms and thus may have a beneficial role in host survival, apoptosis of cells involved in the immune response can favor microbial evasion and opportunistic infections. It has been demonstrated that blocking lymphocyte apoptosis during experimental peritonitis $(2,3)$ and sepsis (4) improved animal survival. In the past decade it has been demonstrated both in vitro (5) and in vivo (6) that microbes or their products are capable of inducing apoptosis in endothelial cells but its impact on the outcome of the disease remains to be determined.

Pseudomonas aeruginosa is a common opportunistic agent of life-threatening sepsis in immunocompromised patients. Studies carried out in our laboratory have demonstrated the ability of different $P$. aeruginosa isolates to induce apoptosis in endothelial cells $(7,8)$. The infected cells produce high amounts of reactive oxygen intermediates and nitric oxide (NO) and cell pre-treatment with antioxidants significantly reduces the percentage of cell death, suggesting a role for free radicals as regulators of apoptosis. Indeed, there is now a growing body of evidence showing that over-generation of oxidant radicals is involved in apoptotic pathways triggered by different stimuli $(9,10)$.

Another major mechanism of apoptosis induction is the activation of the Fas/Fas ligand (Fas-L) pathway, which requires the cross-linking of membrane-bound Fas (mFas) with either cells expressing Fas-L or aggregates of soluble Fas-L (sFas-L) proteolytically cleaved by metalloproteinases from cell surfaces $(11,12)$. Recent studies have not only shown that there is an up-regulation of mFas and Fas- $\mathrm{L}$ on $P$. aeruginosa-infected epithelial cells but also demonstrated a role for this pathway in the apoptotic signaling cascade triggered by $P$. aeruginosa infection (13-16). However, the mechanisms leading to Fas/FasL overexpression during the infection remain unknown.

A number of bacterial factors have been implicated in $P$. aeruginosa pathogenesis. Prominent among these virulence determinants are the toxins translocated directly into eukaryotic 
cells via type III secretion system (TTSS). To date, four toxins have been characterized, including the ADP-ribosylating ExoS and ExoT, an acute cytolytic factor, ExoU, and an adenylate cyclase, ExoY (17). Expression of these effector molecules as well as of the components of the type III secretory apparatus is under the control of a transcriptional activator, ExsA, which responds to cell contact signals (18).

$P$. aeruginosa TTSS proteins have already been reported to induce apoptosis of human cells $(19,20)$ but different cell types exhibited differential levels of sensitivity to TTSS effectors $(21,22)$. Therefore, in this study we examined the role of TTSS proteins and of the Fas/FasL system and in the induction of apoptosis in $P$. aeruginosa-infected endothelial cells. To this end, we compared the effects of infection with the wild-type $P$. aeruginosa PAO1 strain with its isogenic mutant defective in ExsA on human umbilical vein endothelial cells (HUVEC).

\section{Materials and methods}

Bacteria. The laboratory $P$. aeruginosa strains PAO-1, which synthesizes ExoS, ExoT and ExoY, and PAO-1::exsA, defective in all TTSS proteins, were grown in trypticase soy broth. The culture medium of PAO-1::exsA, constructed by plasmid insertion in the exsA gene (23), was supplemented with carbenicilin at $300 \mu \mathrm{g} / \mathrm{ml}$. After incubation for $16-18 \mathrm{~h}$ at $37^{\circ} \mathrm{C}$, bacteria were harvested by centrifugation and resuspended in M-199 medium containing $25 \mathrm{mM}$ HEPES (N,2 hydroxyethylpiperazine-N'2 ethanesulphonic acid), 10\% fetal calf serum and glutamine to an absorbance of 0.1 at $660 \mathrm{~nm}$, which corresponds to $10^{8}$ colony forming units $/ \mathrm{ml}$.

Cell culture. Human umbilical vein endothelial cells (HUVEC) were isolated as described (24) and seeded in cell culture flasks coated with $1 \%$ gelatin in M-199 medium containing $10 \%$ fetal calf serum, glutamine and antibiotics (complete culture medium). Confluent primary cultures were trypsinized, cells were resuspended in complete culture medium, seeded in 96-well $\left(2.5 \times 10^{4}\right.$ cells/well $), 24-w e l l ~\left(2.5 \times 10^{5}\right.$ cells/well $)$ or 6 -well $\left(7.5 \times 10^{5}\right.$ cells/well $)$ tissue culture plates, and cultured for $48 \mathrm{~h}$.

Analysis of the cytotoxic effect of P. aeruginosa TTSS proteins. The effect of $P$. aeruginosa infection on HUVEC cultures was assessed by the methyltiazole tetrazolium (MTT) reduction assay (25). Confluent monolayers of cells cultured in 96-well plates were exposed to PAO-1, PAO-1::exsA or culture medium only (control cells) for $1 \mathrm{~h}$ at $37^{\circ} \mathrm{C}$, rinsed and treated with gentamicin at $300 \mu \mathrm{g} / \mathrm{ml}$ in complete culture medium. After $24 \mathrm{~h}$, cells were rinsed and incubated with MTT at $1 \mathrm{mg} /$ $\mathrm{ml}$ for $1 \mathrm{~h}$. Formazan crystals formed in viable and metabolically active cells were solubilized with isopropanol and the absorbances at $570 \mathrm{~nm}\left(\mathrm{~A}_{570 \mathrm{~nm}}\right)$ were determined using an automatic microplate spectrophotometer. Cell viability in infected cultures was calculated considering the absorbance values of non-infected cultures as $100 \%$.

Flow cytometric analysis of cell reactivity with annexin $V$. Exposure of phosphatidylserine residues at the surface of HUVEC was assessed through flow cytometric analysis of cell labeling with biotinylated annexin V, using the Annexin V-biotin apoptosis detection kit (Roche Molecular Biochemicals, Mannheim, Germany). Cells cultured in 24-well microplates were infected with $P$. aeruginosa strains or exposed to culture medium for $1 \mathrm{~h}$, treated with the gentamicincontaining culture medium for $12 \mathrm{~h}$ and detached by trypsinization. Cell labeling with annexin V was performed according to the manufacturer's instructions. At least 10,000 cells were analyzed with a FACScalibur flow cytometer (Becton Dickinson, Mountain View, CA).

Analysis of DNA fragmentation. DNA fragmentation in infected and non-infected cells cultured in 24-well culture plates was assessed at $12 \mathrm{~h}$ post-infection by photometric detection of histone-associated DNA fragments, using the cell death detection enzyme-linked immunosorbent assay (ELISA; Roche Diagnostic Corp., Mannheim, Germany), according to the manufacturer's instructions. To ensure that the quantitative ELISA was actually measuring apoptosis and not background DNA fragmentation resulting from necrosis, control cells heated at $60^{\circ} \mathrm{C}$ for $30 \mathrm{~min}$ were included in all assays (data not shown).

Detection of membrane-bound Fas and Fas-L by flow cytometry. HUVEC cultured in 24-well microplates were infected with the different $P$. aeruginosa strains for 30 and $60 \mathrm{~min}$. Cells were then rinsed twice with PBS and detached from the microplate wells with $0.05 \%$ EDTA. Fas expression was detected as described by Suhara et al (26). Briefly, infected and control non-infected cells were exposed to $0.5 \mu \mathrm{g} / \mathrm{ml}$ goat polyclonal anti-Fas or anti-FasL IgG (Santa Cruz Biotecnology, Inc.) for $1 \mathrm{~h}$ at $4^{\circ} \mathrm{C}$, rinsed and treated with biotinylated anti-sheep/goat IgG (Amersham). After $1 \mathrm{~h}$ at $4^{\circ} \mathrm{C}$, cells were rinsed and labeled with a streptavidin-FITC complex (Amersham) at $5 \mu \mathrm{g} / \mathrm{ml}$ for $20 \mathrm{~min}$ at $4^{\circ} \mathrm{C}$. Cells were harvested by centrifugation and the pellets were ressuspended in phosphate buffer saline $\mathrm{pH} 7.2$ (PBS) containing $1 \%$ bovine serum albumin for analysis of 10,000 cells by flow cytometry.

Detection of Fas and Fas L mRNA by RT-PCR. Total RNA was isolated from non-infected (control) or from HUVEC infected for 30 min using Qiagen Rneasy kit. cDNA was synthesized from $2 \mu \mathrm{g}$ of total RNA by reverse transcription with the SuperScript $^{\mathrm{TM}}$ First-Strand synthesis system for RT-PCR (Invitrogen) according to the manufacturer. cDNA was diluted (1/10) and $1 \mu 1$ was subjected to the following PCR conditions: denaturation at $95^{\circ} \mathrm{C}$ for $5 \mathrm{~min}$ and 35 cycles of denaturation at $95^{\circ} \mathrm{C}$ for $45 \mathrm{sec}$, annealing at $58^{\circ} \mathrm{C}$ for $1 \mathrm{~min}$, and extension at $72^{\circ} \mathrm{C}$ for $1 \mathrm{~min}$. An additional extension step of $5 \mathrm{~min}$ at $72^{\circ} \mathrm{C}$ was carried out after the last cycle. The primers used in the reactions were: 5'-ATG CTG GGC ATC TGG ACC CT-3' (Fas sense); 5'-CAA CAT CAG ATA AAT TTA TTG CCA C-3' (Fas antisense); 5'-GGA TGT TTC AGC TCT TCC AC-3' (Fas L sense); 5'-TCT TCC CCT CCA TCA TCA CC-3' (Fas L antisense), 5'-CCT CGC CTT TGC CGA TCC-3' (B-actin sense) and 5'-GGA TCT TCA TGA GGT AGT CAG TC-3' ( $B$-actin antisense). PCR products were subjected to electrophoresis in a $2 \%$ agarose gel and stained with ethidium bromide. Densitometry was performed using the LabImage software (Kaplan GmbH, Germany). 
Detection of sFas L. HUVEC cultured in 6-well microplates were infected with PAO-1 and PAO-1::exsA for $1 \mathrm{~h}$, rinsed and incubated with the gentamicin-containing culture medium for an additional $12 \mathrm{~h}$. The supernatants from cultures infected with each bacterial strain $(10 \mathrm{ml})$ were concentrated to $100 \mu \mathrm{l}$ and sFasL was measured by ELISA (Quantikine, RD systems), according to the manufacturer's instructions. Cell supernatants were also analyzed by SDS-PAGE (30 $\mu \mathrm{g}$ for each lane) on $10 \%$ polyacrylamide gels. Proteins were transferred to PVDF membranes (Millipore) and replicas were incubated overnight at $4{ }^{\circ} \mathrm{C}$ with PBS containing $2 \%$ BSA and $0.05 \%$ Tween-20, washed and incubated with goat IgG antihuman Fas L (Santa Cruz Biotecnology, Inc.) for $1 \mathrm{~h}$ at room temperature. Membranes were then washed, incubated with biotinylated anti-sheep/goat $\operatorname{IgG}$, washed and incubated with a horseradish peroxidase-conjugated streptavidin (Amersham). After washing, FasL was detected using an enhanced chemiluminescence kit (ECL; Amersham). Densitometry was performed using Scion Image software (Scion, USA).

Analysis of the effect of an antagonistic anti-Fas on cell reactivity with annexin $V$. Cells cultured in 24-well microplates were infected with the $P$. aeruginosa strains and co-treated with the antagonistic anti-Fas $\operatorname{IgG}_{3}(10 \mu \mathrm{g} / \mathrm{ml}$; Santa Cruz $)$. After $1 \mathrm{~h}$, cells were rinsed and treated with culture medium containing gentamicin and anti-Fas $\mathrm{IgG}_{3}$. Control non-infected cells were submitted to the same procedure. After $12 \mathrm{~h}$, cells were trypsinized and labeled with annexin V, as described above. At least 10,000 cells were analyzed by flow cytometry.

Analysis of the effect of LNMMA on cell reactivity with annexin $V$. Cells cultured in 24-well microplates pre-treated for $24 \mathrm{~h}$ with $200 \mathrm{mM}$ LNMMA (Sigma) were exposed to the $P$. aeruginosa strains or culture medium, in the presence of LNMMA. After $1 \mathrm{~h}$, cells were rinsed and treated with culture medium containing gentamicin and LNMMA for $12 \mathrm{~h}$. Cells were detached by trypsinization and labeled with annexin $\mathrm{V}$ for analysis of 10,000 events by flow cytometry, as described.

Analysis of NO production. NO reacts with water in culture medium to produce nitrite, whose concentration can be taken as a measure of NO production. The nitrite concentration in the supernatants of cells infected for $12 \mathrm{~h}$ and of non-infected cells was determined by the Griess reaction, as previously described (8).

Detection of the inducible isoform of NO-synthesizing enzyme (iNOS). Cells were infected with PAO-1 and PAO-1::exsA for $1 \mathrm{~h}$, treated with gentamicin-containing medium for an additional $1 \mathrm{~h}$, rinsed twice with PBS, detached from culture plates by tripsinization, fixed with $4 \%$ paraformaldehyde and $4 \%$ sucrose in PBS for 20 min and permeabilized with $0.1 \%$ Triton X-100 for $5 \mathrm{~min}$. Cells were then rinsed, centrifuged, and the pellets were resuspended in PBS containing 5\% fetal calf serum and polyclonal rabbit anti-iNOS IgG (Santa Cruz Biotecnology, Inc.) at $2 \mu \mathrm{g} / \mathrm{ml}$. After $1 \mathrm{~h}$ at $4^{\circ} \mathrm{C}$, cells were rinsed, incubated with FITC-conjugated anti-rabbit IgG for $1 \mathrm{~h}$ at $4^{\circ} \mathrm{C}$ and harvested by centrifugation. Finally, pellets were resuspended in PBS containing $1 \%$ bovine serum albumin and submitted to flow cytometry analysis.
Statistical analysis. Results are expressed as means \pm SEM of data obtained in at least three different experiments. Statistical differences between groups were determined with a one-way analysis of variance (ANOVA) followed by the Dunnett's or Bonferroni's multiple comparison post test or with the MannWhitney U test. Statistical significance was set at $\mathrm{p}<0.05$.

\section{Results}

P. aeruginosa TTSS enhanced apoptosis in infected endothelial cells. To gain insight into the effects of TTSS proteins on host cells, HUVEC were exposed to $P$. aeruginosa PAO-1 or to the defective strain PAO-1::exsA. After $1 \mathrm{~h}$, cells were treated with gentamicin-containing culture medium for different periods to eliminate extracellular bacteria. Cell death induced by infection was assessed by the MTT reduction assay whereas apoptosis was characterized by DNA fragmentation (26) and cell reactivity with annexin V (27). As shown in Fig. 1A, the wild-type strain was significantly more cytotoxic than the TTSS-deficient mutant. Fig. 1B shows the results of the quantification of cytoplasmic DNA fragments in control non-infected and in infected cells by ELISA. Although both strains induced increased cleavage of DNA, fragmentation was significantly higher $(\mathrm{p}<0.001)$ in cells infected with the wild-type bacteria.

In normal viable cells, phosphatidylserine is located on the cytoplasmic leaflet of the cell membranes whereas in apoptotic cells phosphatidylserine is exposed on cell surfaces. In vitro, interaction of the externalized PS with FITC-labelled annexin V allows the detection and quantification of apoptotic cells (27). Fig. 1C shows that the mean percentage of annexin V-reactive cells were significantly higher $(\mathrm{p}<0.05)$ in cultures infected with the parental bacteria when compared with the cultures infected with the mutant. In Fig. 1D, a representative FACS analysis shows that annexin V-labelled cells did not incorporate propidium iodide, once more demonstrating that the cell death observed displays features of apoptosis.

$P$. aeruginosa TTSS up-regulated Fas and Fas-L expression in HUVEC. Fas and FasL are stored in cell cytoplasm from where they traffic to the cell surface (28). Up-regulation of cell surface expression of Fas and Fas-L has been shown to be pivotal for induction of apoptosis in epithelial cells infected by several $P$. aeruginosa strains (13-15). Therefore, we investigated whether $P$. aeruginosa would also modulate the expression of surface Fas and FasL in HUVEC and the role of TTSS in the up-regulation of these proteins. As shown in Fig. 2A, PAO-1 infection triggered a significant increase in the surface expression of Fas in infected cells at 30 and 60 min post-infection $(p<0.001)$. In contrast, cells infected with the TTSS mutant did not differ from non-infected cultures. On the other hand, semi-quantitative RT-PCR analysis showed that neither PAO-1 nor PAO-1::exsA were able to alter the expression of Fas mRNA (Fig. 2B), suggesting that the enhanced expression of $\mathrm{mFas}$ is due to translocation of stored protein rather than to protein neosynthesis. Fig. 2C shows that infection with both bacterial strains led to an increased percentage of cells expressing Fas-L after 30 and $60 \mathrm{~min}$. Although the percentage of cells infected with the wild-type bacteria exhibiting membrane-bound Fas-L was higher than 

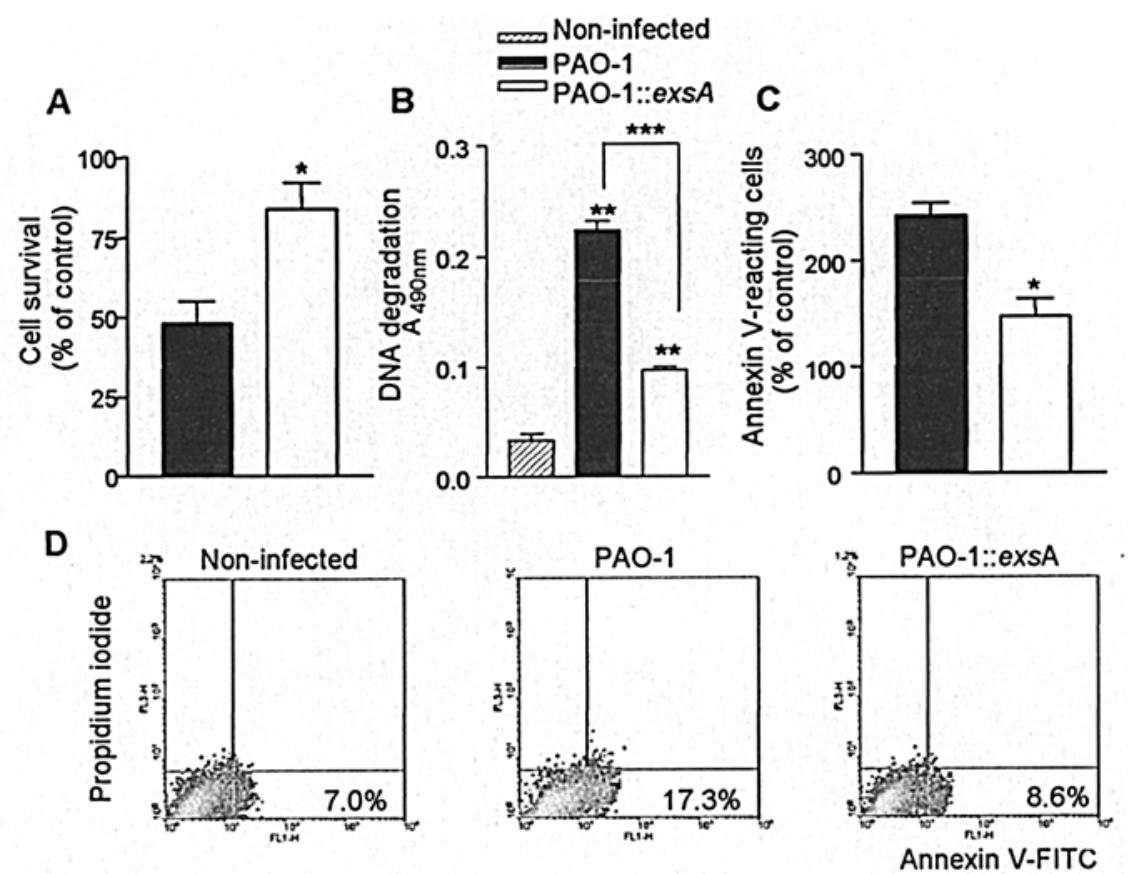

Figure 1. Effect of functional $P$. aeruginosa TTSS system on HUVEC killing. (A and C) The results obtained with control non-infected cultures were set as $100 \%$. (A) Cell viability in cultures infected with the TTSS-producing PAO-1 strain and the TTSS defective mutant PAO-1::exsA, assessed by the MTT reduction assay at $24 \mathrm{~h}$ post-infection. (B) ELISA detection of DNA fragments in lysates of infected and non-infected cells at $12 \mathrm{~h}$ post-infection. (C) Breakdown of phosphatidylserine asymmetry in HUVEC plasma membranes at $12 \mathrm{~h}$ post-infection assessed by cell labeling with annexin V-FITC. In each case, data represent mean values \pm SEM of the results obtained in at least three independent experiments carried out in triplicate. ${ }^{*} \mathrm{p}<0.05$ and ${ }^{* * *} \mathrm{p}<0.001$ when data obtained from PAO-1 infected cells were compared with those from PAO-1::exsA-infected cultures. (D) Representative histograms of 1 out of 5 experiments of cell labeling with annexin V-FITC.



Figure 2. Effect of TTSS proteins on Fas (A) and Fas-L (C) expression by infected and control non-infected cells, assessed by FACS analysis. Data are mean values \pm SEM of the results obtained in three assays. (A) ${ }^{* *} \mathrm{p}<0.01$ when the results obtained from PAO-1-infected cells were compared with those from noninfected or PAO-1::exsA-infected cells. (C) ${ }^{*} \mathrm{p}<0.05$ and ${ }^{* *} \mathrm{p}<0.01$ when data obtained from infected cultures were compared with those from control noninfected cultures. Effect of P. aeruginosa infection on Fas (B) and Fas-L (D) mRNA. PAO-1 up-regulated Fas-L mRNA expression.

that detected in PAO-1::exsA-infected-cultures (Fig. 2C), the differences were not statistically significant. In contrast, these differences were evident at the transcription level since RTPCR analysis showed that PAO-1 up-regulated Fas-L mRNA expression when compared with non-infected cells while PAO-1::exsA failed to do so (Fig. 2D).
$P$. aeruginosa TTSS also up-regulated the release of soluble Fas- $L$. Fas-L is expressed as a $40 \mathrm{kDa}$ membrane-bound protein and can be cleaved by matrix metalloproteinases to release a $28-\mathrm{kDa}$ soluble protein (sFas-L) (29). Since triggering of the apoptotic signaling by the Fas pathway requires the cross-linking of Fas either with cells expressing 

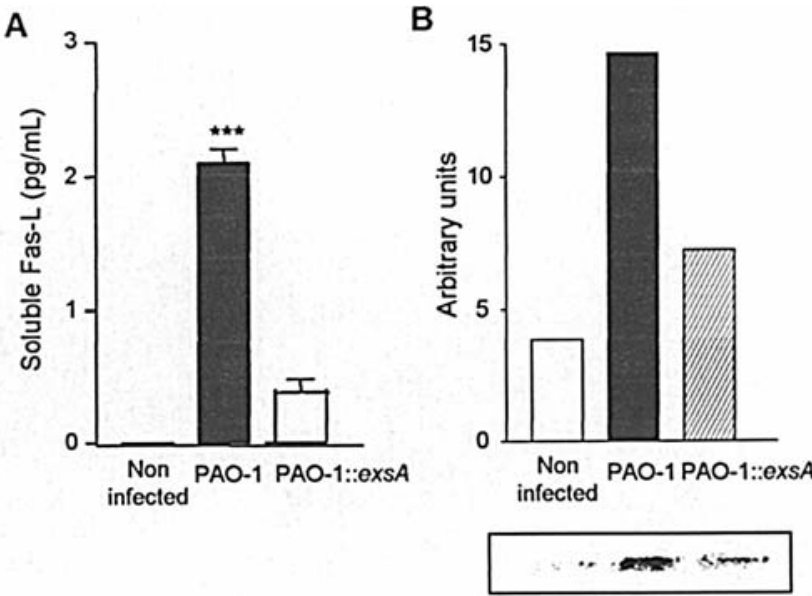

Figure 3. (A) Soluble Fas-L concentration in supernatant of HUVEC infected for $12 \mathrm{~h}$ with $P$. aeruginosa, as determined by ELISA. Data are means \pm SEM of two experiments carried out in duplicate. ${ }^{* * *} \mathrm{p}<0.001$ when cells infected with PAO-1 were compared with cells infected with PAO-1::exsA. (B) Western blot analysis of sFas-L in supernatants from infected and control non-infected cells. Densitometry confirmed the differences between the levels of sFas-L in the diverse culture supernatants.

membrane-bound Fas-L or with sFas-L (30), we next investigated the presence of sFas-L in the supernatants of infected cells by ELISA and immunoblotting. As shown in Fig. 3A and B, the release of sFas-L from PAO-1-infected HUVEC was significantly higher $(p<0.001)$ than that observed in cultures infected with the ExsA-defective mutant.

Anti-human Fas significantly reduced but did not completely abrogate apoptosis of infected HUVEC. Our results showing that cultures infected with the TTSS-producing PAO-1 strain exhibited higher percentage of Fas-expressing cells and released more sFas-L led us to investigate the role of the Fas/ Fas-L pathway in HUVEC apoptosis. To this end, cells were treated or not with an antagonist antibody (IgG anti-Fas), that blocks the interaction between $\mathrm{mFas}$ and $\mathrm{sFas}-\mathrm{L}$, prior to and during the infection period. The percentage of apoptotic cells in treated and untreated infected cultures was then determined by the annexin $\mathrm{V}$ assay. As shown in Fig. 4A, treatment with the neutralizing antibody significantly reduced the percentage of apoptotic cells in the cultures infected with the parental bacteria, but not in cultures infected with the TTSS-defective mutant. However, neutralization of membrane-bound Fas with the antagonist antibody did not completely abrogate apoptosis of cells infected with the PAO-1 strain. Thus, our results demonstrate that bacteria exhibiting a functional TTSS induce apoptosis in HUVEC by upregulating the Fas/Fas-L system and even more by an additional and yet unknown mechanism. Fig. 4B shows representative histograms obtained in 1 out of 3 analysis of cell labeling with annexin V-FITC.

NO produced in response to $P$. aeruginosa infection is the major mechanism accounting for HUVEC apoptosis. Our group and others have previously shown that endothelial cells produce high amounts of NO in response to bacterial infection which ultimately leads to cell death (8,31-33). Therefore, we investigated the participation of NO in HUVEC apoptosis induced by $P$. aeruginosa TTSS by comparing the apoptosis
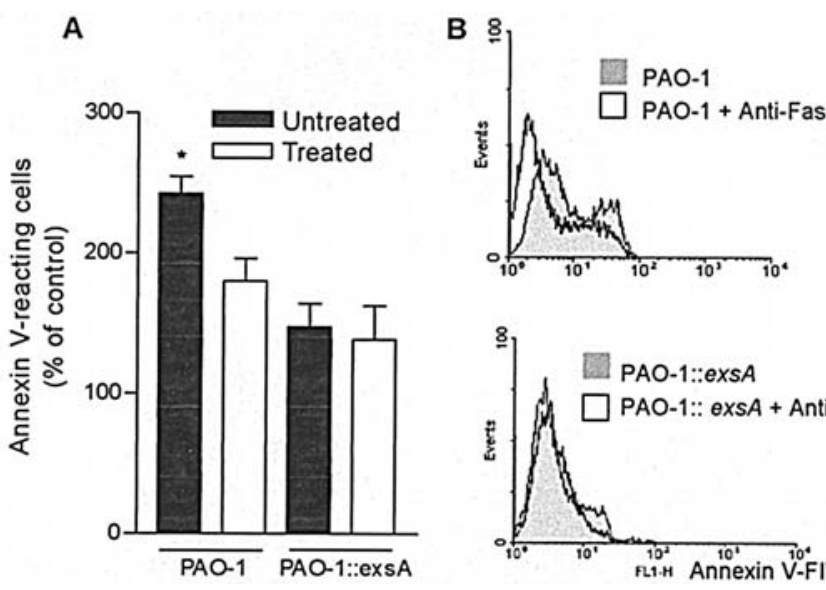

Figure 4. Effect of anti-Fas blocking $\mathrm{IgG}$ on apoptosis of $P$. aeruginosainfected HUVEC, determined by FACS analysis of cell labeling with annexinV-FITC. (A) Data represent mean values \pm SEM of the results obtained with infected cells, setting the percentage of positive cells obtained with control non-infected cells as $100 \%$, as determined in four assays. * $\mathrm{p}<0.05$ when data obtained from untreated PAO-1-infected cells were compared with IgG-treated PAO-1-infected cells or untreated PAO-1::exsAinfected cells. (B) Representative histograms obtained after cell labeling with annexin V-FITC.

rates in infected cultures previously treated with LNMMA, a potent inhibitor of NO synthesis, with that in untreated infected cultures. Fig. 5 shows that the inhibition of NO synthesis by LNMMA resulted in significant decrease $(p<0.001)$ in the percentage of annexin V-reactive cells in both PAO-1 and PAO-1::exsA infected cultures to levels similar to that observed in control non-infected cells. Fig. 5B shows representative histograms of cell labeling with annexin V-FITC.

TTSS up-regulated NO production and iNOS expression in $H U V E C$. To fit our hypothesis that enhanced production of NO was directly involved in endothelial cell apoptosis with the finding of a causal relationship between a functional TTSS and apoptosis of infected HUVEC (Figs. 1 and 4), we investigated whether PAO-1 and PAO-1::exsA strains induced different levels of NO production. Fig. 6A and B inequivocally show that only the TTSS-producing bacteria markedly enhanced the HUVEC generation of NO by up-regulating iNOS, known to generate high concentrations of NO for prolonged periods (34).

NO, and not TTSS, directly up-regulated Fas expression in HUVEC. Different studies have shown that high levels of $\mathrm{NO}$ and related reactive nitrogen species may trigger the upregulation of Fas and Fas-L $(35,36)$. Therefore, we asked, whether the increase of Fas and Fas-L detected in cells infected with PAO-1 had resulted directly from the enhancement of NO generation, rather than from the presence of a functional TTSS. To address this question, the expression of membrane-bound Fas and Fas-L in PAO-1 infected cells treated and untreated with LNMMA was investigated. Fig. 7A shows that cell treatment decreased significantly $(\mathrm{p}<0.05)$ the percentage of Fas-expressing cells to levels similar to those exhibited by control non-infected cultures. Cell treatment also decreased the percentage of Fas-L-expressing cells but the difference was not significant (Fig. 7B). Fig. 7C and D show representative 

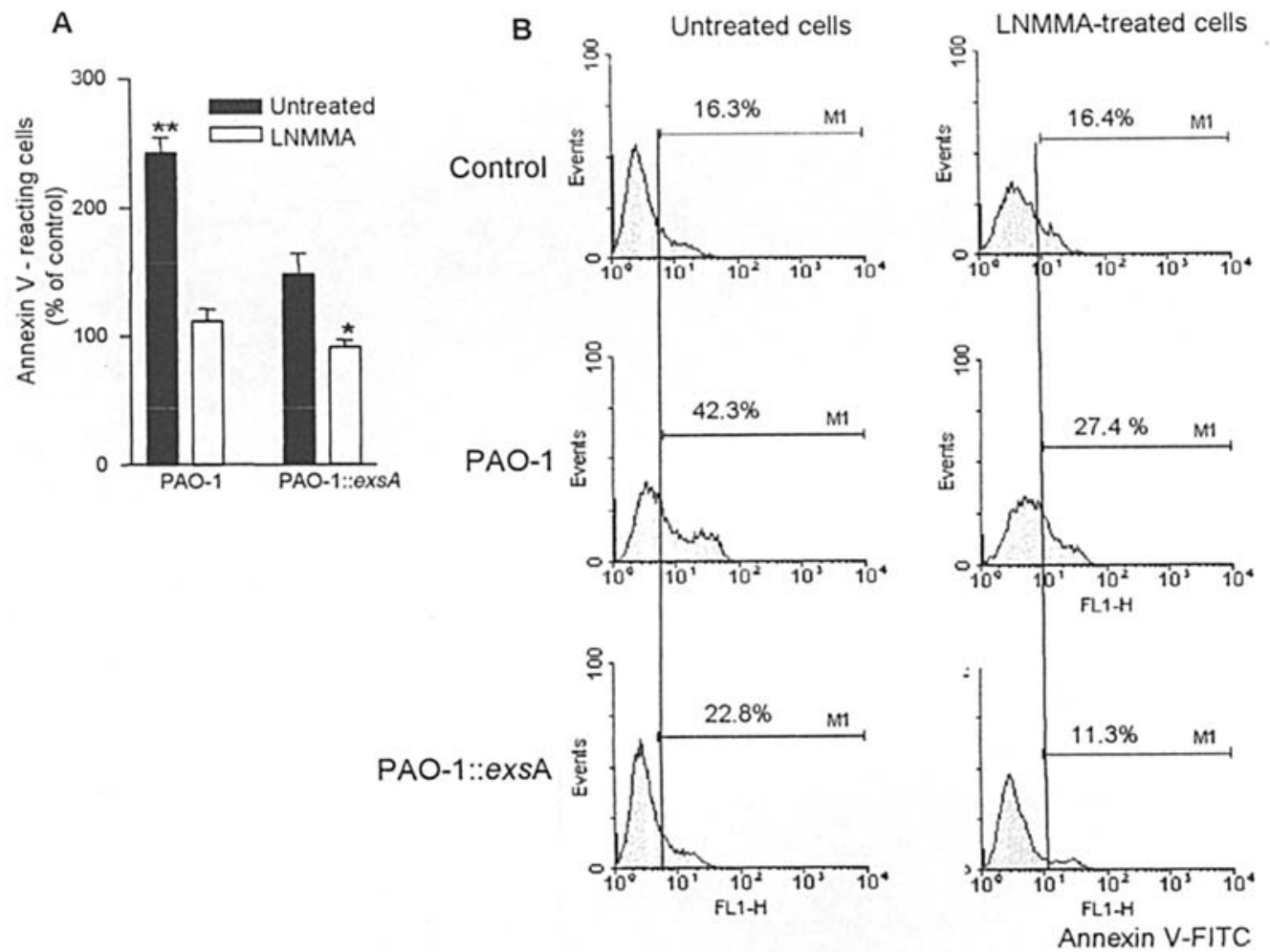

Figure 5. (A) Effect of LNMMA on apoptosis of P. aeruginosa infected HUVEC, assessed by FACS analysis of cell labeling with annexin V-FITC, setting the labeling of control non-infected cells as $100 \%$. Results are means \pm SEM obtained in four assays in which 10,000 cells were analyzed. ${ }^{* *}$ p $<0.01$ when data obtained with untreated PAO1-infected cells were compared with those from LNMMA-treated PAO1-infected cells or untreated PAO-1::exsA-infected cells. " $\mathrm{p}<0.5$ when data obtained with untreated PAO1::exsA-infected cells were compared with those from LNMMA-treated PAO1::exsA-infected cells. (B) Representative histograms of 1 out of 4 experiments of cell labeling with annexin V-FITC.

histograms of the effect of LNMMA treatment on cell labeling with anti-Fas and anti-Fas-L, respectively.

\section{Discussion}

$P$. aeruginosa TTSS has been reported to induce apoptosis in several cell types (37-41), but the mechanisms regulating cell death are not completely understood. Early studies from Jendrossek et al (14) indicated a TTSS-dependent bacterial activation of two distinct proapoptotic signaling pathways in infected epithelial cells. The first depends on the induction of mitochondrial alterations, including depolarization and release of cytochrome c, whereas the second mainly involves the stimulation of Jun N-terminal kinases (JNK). Both mitochondrial changes and JNK activation were likely initiated by Fas-Fas-L interactions as a result of cell surface up-regulation of the receptor-ligand pair upon infection. Similarly, others have described increased expression of Fas and Fas-L in $P$. aeruginosa-infected epithelial cells and subsequent apoptosis $(13,16,42)$. However, no previous study has highlighted the mechanisms regulating the overexpression of these proteins.

Herein, we demonstrated the induction of apoptosis of human endothelial cells by the TTSS producing PAO-1 strain. At a first glance this conclusion may seem contradictory to our previous report that this same $P$. aeruginosa strain induces endothelial cell death by a mechanism distinct from apoptosis (23). However, infection conditions are known to modulate the response of host cells to infecting micro-organisms. Whereas in our former study cells were kept in contact with high concentrations of bacteria for $3 \mathrm{~h}$, in the present study cell cultures were exposed to bacteria for $1 \mathrm{~h}$ and then treated with an antibiotic-containing culture medium for additional periods, to eliminate the effect of multiplying extracellular microorganisms. With this approach, we showed that besides inducing HUVEC apoptosis, PAO-1 also up-regulated the expression of Fas and Fas-L, as well as the release of sFas-L from infected cells. In contrast, cells infected with the isogenic TTSS defective mutant failed to induce a significant up-regulation of Fas and induced only a mild release of sFas-L. It would be tempting to conclude that cell apoptosis was dependent upon Fas-Fas-Lmediated signaling pathway triggered by bacterial TTSS effectors. However, cells infected with the TTSS mutant exhibited characteristics of apoptosis as well, although less frequently. This finding suggests that, in addition to a functional TTSS, other bacterial virulence mechanisms present both in wild-type and mutant bacteria are involved in endothelial cell death. This hypothesis is consistent with earlier reports from Yamada et al (43) and Goto et al (44) in which the authors demonstrate a role for PAO-1 $P$. aeruginosa redox protein azurin in macrophage apoptosis through stabilization of the tumor suppressor protein $\mathrm{p} 53$. Moreover, subsequent studies carried out by Jendrossek et al (15) showed that type IV pili is also involved in apoptosis of infected epithelial cells, through a mechanism involving Fas up-regulation and subsequent caspase- 8 and 3 activation.

Indirect evidences suggest that the Fas-Fas-L signaling pathway may play a role in modulating the apoptotic cascades 
A

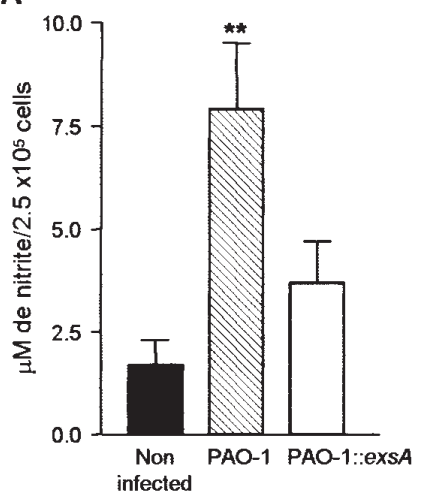

B



Figure 6. (A) Nitrite concentration detected in supernatants from cells infected for $12 \mathrm{~h}$ and from control non-infected cells. Data are means \pm SEM of five assays. ${ }^{* *} \mathrm{p}<0.01$ when the results obtained in cultures infected with the wild-type bacteria were compared with those from control cells and from cells infected with the mutant. (B) FACS detection of iNOS expression by cells infected for $2 \mathrm{~h}$ and by control non-infected HUVEC. Data represent means \pm SEM of the median fluorescence intensity obtained in three independent assays. ${ }^{* *} \mathrm{p}<0.01$ when data obtained from PAO-1-infected cells were compared with those from control or PAO-1::exsA-infected cells.

during viral, bacterial and parasitic infections (45). Concerning the role played by this signaling pathway in apoptosis of $P$. aeruginosa-infected endothelial cells, we observed that despite the enhancement of mFas expression in cells exposed to the wild-type bacteria, cell treatment with an antagonist anti-Fas IgG inhibited apoptosis only partially. Therefore, although an autocrine loop of Fas and sFas-L may be involved in apoptosis of $P$. aeruginosa-infected HUVEC, our findings argue against a critical role for the Fas signaling pathway, at least in our experimental model. However, it should be emphasized that in vivo, the induction of Fas expression by infected endothelial cells is likely to be relevant by sensitizing infected cells to apoptosis triggered by FasL-expressing inflammatory cells. Moreover, it has been suggested that Fas may transduce cell activation signals independently of the cell death pathway (46). Fas activation in tissue endothelial cells has been shown to result in the secretion of chemotatic factors resulting in amplified recruitment of leukocytes and tissue injury (46).

Another important finding of our study was the massive release of sFasL from cells infected with the TTSS-producing bacteria. Although native sFasL normally does not activate Fas (47), and has even been reported to inhibit apoptosis $(48,49)$, secondarily aggregated $\mathrm{sFasL}$ is sufficient to induce Fas signaling and induce apoptosis $(47,50)$. However, the precise role played by sFasL release in the course of $P$. aeruginosa infection, as well as the role of the TTSS effectors in sFasL release, still awaits further investigation.

In addition to the partial role played by TTSS in the induction of apoptosis through the Fas-Fas-L signaling pathway, the results demonstrated for the first time a $P$. aeruginosa TTSS-dependent overexpression of iNOS resulting in high output NO production, which in turn plays a critical role in the induction of apoptosis.

NO is an important intracellular and intercellular signaling molecule involved in the regulation of a number of physiological and pathological processes, with potent proinflammatory, immunomodulatory and antimicrobial activities (34).

A
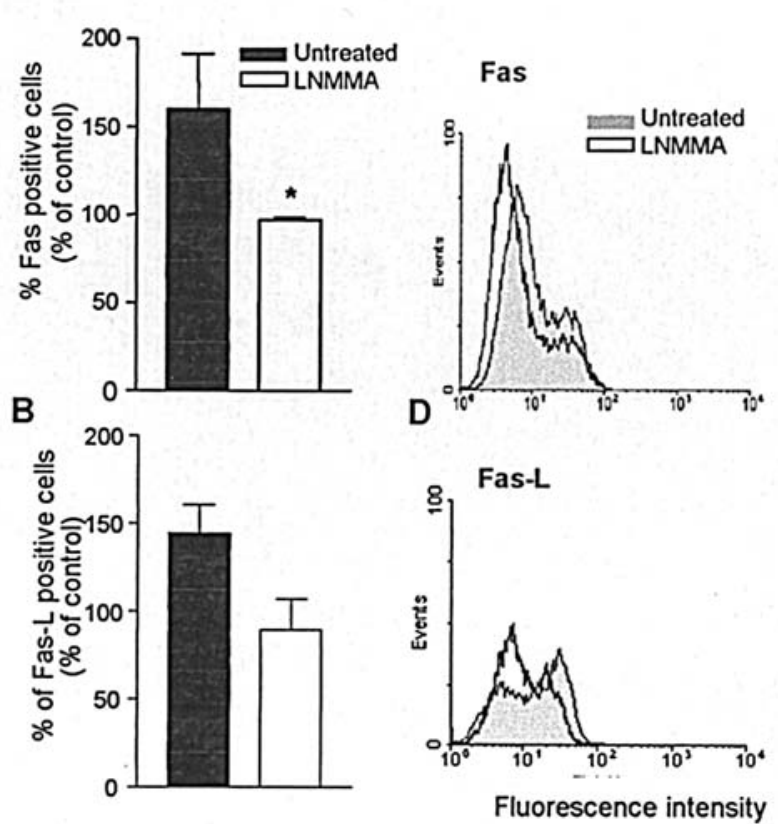

Figure 7. (A) Effect of LNMMA on Fas expression by cells infected with PAO-1 for $1 \mathrm{~h}$, assessed by FACS analysis. Data represent mean values \pm SEM of results obtained with treated and untreated cells, setting the labeling of control non-infected cells as $100 \%$, as determined in four assays. ${ }^{*} \mathrm{p}<0.05$. $\mathrm{B}$ and $\mathrm{C}$ show representative histograms of experiments of LNMMAtreated and untreated cell labelling with anti-Fas (B) or with anti-Fas-L (C) antibodies.

Within endothelial cells, NO is produced through enzymatic oxidation of L-arginine by the constitutively expressed endothelial NO synthase (eNOS), which synthesize intermittent low levels of NO involved in the regulation of blood vessel tone in the vascular system, and by the inducible isoform of the enzyme (iNOS) that, once expressed, generates high concentrations of NO. Although in phagocytic cells activated iNOS contributes to host defense by generating a potent defense molecule against microbial pathogens, oxidation products of high-output NO production can be highly cytotoxic and exert pro-apoptotic activities by different pathways, leading to cell death $(51,52)$.

A major activator of iNOS is bacterial lipopolysaccharide (34). Like several other microorganisms, P. aeruginosa has been noted to increase the expression of iNOS in mammalian cells $(8,53)$, although the mechanism of induction has not been worked out in detail. Our finding that the levels of iNOS were similar in cells infected with the bacteria defective in TTSS and in control non-infected cells suggests the involvement of TTSS effector proteins in iNOS induction, similarly to that observed for Salmonella Sips B, C, and D and SopE2 in infected macrophages (54). However, the findings do not exclude a role for LPS in this process.

Recent studies have shown that NO production may be closely related to the signaling pathways that regulate the polymerization of actin (55) and microtubule cytoskeleton (56), since disruption of actin enhances both iNOS mRNA and protein expression in endothelial cells via transcriptional regulation $(57,58)$. PAO-1 P. aeruginosa is known to synthesize ExoS and ExoT, two TTSS effector proteins that disrupt actin cytoskeleton with their N-terminal GTPase- 
activating protein domain, resulting in cell rounding and detachment (59-61). The identification of the precise TTSS effector protein possibly involved in the regulation of iNOS was beyond the focus of this study but further studies employing different $P$. aeruginosa mutants will be carried out to clarify this question. Bacterial regulation of iNOS by modifying the host cell cytoskeleton is a novel concept that may be a step forward in our understanding of the pathophysiology of $P$. aeruginosa infection.

We demonstrated that, besides its role in induction of apoptosis, the NO produced by cells infected with the TTSSproducing bacteria contributed to the augmented expression of mFas and release of sFas-L. Our findings are consistent with earlier reports showing a NO-dependent up-regulation of Fas expression in different cell types (36,62-64).

In summary, the results reveal novel molecular details of the interaction between $P$. aeruginosa and human host cells. We demonstrated for the first time that besides accounting for a higher expression of membrane-bound Fas and a higher release of sFas-L, TTSS proteins also contributed for overproduction of NO. Furthermore, the results suggest that, rather than Fas induction, increased NO production is the major mechanism responsible for apoptosis of P. aeruginosainfected HUVEC. It should be stressed that the higher expression of mFas and release of sFas-L by infected endothelial cells may play a pivotal role and represent an event of biological significance in vivo by rendering cells more susceptible to apoptosis induced by Fas-L expressing inflammatory cells. Experiments to address this hypothesis will be a fruitful avenue for further research.

\section{Acknowledgements}

We thank Maria Angélica P. da Silva for her technical assistance. We are also grateful to the personnel from the Hospital Maternidade Carmela Dutra (Rio de Janeiro, Brazil) for providing the umbilical cords. This research was supported by grants from CNPq. C.F. was supported by a scholarship from CNPq.

\section{References}

1. Zychlinsky A and Sansonetti PL: Apoptosis in bacterial pathogenesis. Microb Pathog 17: 203-212, 1994.

2. Ayala A, Hemdom C, Lehman D, Ayala CD and Chandry LH: Differential induction of apoptosis in lymphoid tissues during sepsis; variation in onset, frequency, and the nature of the mediators. Blood 87: 4261-4275, 1986.

3. Hotchkiss RS, Swanson PE, Cobb JP, Jacobson A, Buchman TG and Karl LA: Apoptosis in lymphoid and parenchymal cells during sepsis: findings in norm al and T- and B-cell deficient mice. Crit Care Med 25: 1298-1307, 1997.

4. Grobmyer SR, Armstrong RC, Nicholson SC, Gabay C, Arend WP, Potter SH, Melchior M, Fritz LC and Nathan CF: Peptidomimetic fluoromethylketone rescues mice lethal endotoxic shock. Mol Med 5: 585-594, 1999.

5. Menzies BE and Kourteva L: Internalization of Staphylococcus aureus by endothelial cells induces apoptosis. Infect Immun 66: 431-439, 1998.

6. Haimovitz-Friedman A, Cordon-Cardo C, Bayoumy S, Garzotto M, McLoughlin M and Gallily R: Lipopolysaccharide induces disseminated endothelial apoptosis requiring ceramide generation. J Exp Med 186: 1831-1841, 1997.

7. Valente E, Assis MC, Alvim IMP, Pereira IGMB and Plotkowski MC: Pseudomonas aeruginosa induces apoptosis in human endothelial cells. Microb Pathog 29: 345-356, 2000 .
8. Assis MC, Saliba AM, Vidipó LA, Salles J and Plotkowski MC: Pseudomonas aeruginosa-induced production of free radicals by IFN $\gamma$ plus $\mathrm{TNF} \alpha$-activated endothelial cells: mechanism of host defense or of bacterial pathogenesis? Immunol Cell Biol 82: 383-392, 2004.

9. Buttke TM and Sandstrom PA: Oxidative stress as a mediator of apoptosis. Immunol Today 15: 7-10, 1994.

10. Jacobson MD: Reactive oxygen species and programmed cell death. Trends Biochem Sci 21: 83-86, 1996.

11. Schulze-Osthoff K: The Fas/APO-1 receptor and its deadly ligand. Trends Cell Biol 4: 421-426, 1994.

12. Henkler F, Behrle E, Dennehy KM, Wicovsky A, Peters N and Warnke C: The extracellular domains of FasL and Fas are sufficient for the formation of supramolecular FasL-Fas clusters of high stability. J Cell Biol 168: 1087-1098, 2005.

13. Grassmé H, Kirschnek S, Riethmueller J, Riehle A, von Kurthy G and Lang F: Host defense to Pseudomonas aeruginosa requires CD95/CD95 ligand interactions on epithelial cells. Science 290: 527-530, 2000 .

14. Jendrossek V, Grassmé H, Mueller I, Lang F and Gulbins E: Pseudomonas aeruginosa-induced apoptosis involves mitochondria and stress-activated protein kinases. Infect Immun 69: 2675-2683, 2001

15. Jendrossek V, Fillon S, Belka C, Muller I, Puttkamme B and Lang F: Apoptotic response of Chang cells to infection with Pseudomonas aeruginosa strains PAK and PAO-I: molecular ordering of the apoptosis signaling cascade and role of type IV pili. Infect Immun 71: 2665-2673, 2003.

16. Cannon CL, Kowalski MP, Stopak KS and Pier GB: Pseudomonas aeruginosa-induced apoptosis is defective in respiratory epithelial cells expressing mutant cystic fibrosis transmembrane conductance regulator. Am J Respir Cell Mol Biol 29: 188-197, 2003.

17. Hueck CJ: Type III protein secretion systems in bacterial pathogens of animals and plants. Mol Biol Rev 62: 379-433, 1998.

18. Hovey AK and Frank DW: Analyses of the DNA-binding and transcriptional activation properties of ExsA, the transcriptional activator of the Pseudomonas aeruginosa exoenzyme S regulon. J Bacteriol 177: 4427-4436, 1995.

19. Kudoh I, Wiener-Kronish JP, Hashimoto S, Pittet JF and Frank D: Exoproduct secretions of $P$. aeruginosa strains influence severity of alveolar epithelial injury. Am J Physiol 267: L551-L556, 1994.

20. Fleiszig SMJ, Wiener-Kronish JP, Miyazaki H, Vallas V, Mostov KE and Kanada D: Pseudomonas aeruginosa-mediated cytotoxicity and invasion correlate with distinct genotypes at the loci encoding exoenzyme S. Infect Immun 65: 579-586, 1997.

21. Coburn J and Frank DW: Macrophage and epithelial cells respond differently to the Pseudomonas aeruginosa type III secretion system. Infect Immun 67: 3151-3154, 1999.

22. McGuffie EM, Fraylick JE, Hazen-Martin DJ, Vincent TS and Olson JC: Differential sensitivity of human epithelial cells to Pseudomonas aeruginosa exoenzyme S. Infect Immun 67: 3494-3503, 1999.

23. Saliba AM, Filloux A, Ball G, Silva ASV, Assis MC and Plotkowski MC: Type III secretion-mediated killing of endothelial cells by Pseudomonas aeruginosa. Microb Pathog 33: 153-166, 2002.

24. Plotkowski MC, Saliba AM, Pereira SHM, Cervante MP and Bajolet-Laudinat O: Pseudomonas aeruginosa selective adherence to and entry into human endothelial cells. Infect Immun 62: 5456-5463, 1994.

25. Denizot S and Lange R: Rapid colorimetric assay for cell growth and survival. Modification of the tetrazolium dye procedure giving improved sensibility and reliability. J Immunol Methods 89: 271-277, 1986.

26. Suhara T, Fukuo K, Sugimoto T, Morimoto M, Nakahashi T, Hata S, Shimizu M and Ogihara T: Hydrogen peroxide induces up-regulation of Fas in human endothelial cells. J Immunol 160: 4042-4047, 1998.

27. Koopman G, Reutelingsperger CPM, Kuijten GAM, Keehnen K, Pals ST and van Oers HS: Annexin V for flow cytometric detection of phosphatidylserine expression on B cells undergoing apoptosis. Blood 84: 1415-1420, 1994.

28. Bennett M, MacDonald K, Chan SW, Luzio JP, Simari R and Weissberg P: Cell surface trafficking of FasL a rapid mechanism of p53-mediated apoptosis. Science 282: 290-293, 1998.

29. Kayagaki N, Kawasaki A, Ebata T, Ohmoto H, Ikeda S and Inoue S: Metalloproteinase-mediated release of human Fas ligand. J Exp Med 182: 1777-1783, 1995. 
30. Tanaka M, Itai T, Adachi M and Nagata S: Downregulation of Fas ligand by shedding. Nature Med 4: 31-36, 1998.

31. Silverman DJ and Santucci LA: Potencial for free radicalinduced lipid peroxidation as a cause of endothelial cell injury in Rocky Moutain spotted fever. Infect Immun 56: 3110-3115, 1998.

32. Hong Y, Santucci LA, Tian X and Silverman DJ: Superoxide dismutase-dependent, catalase-sensitive peroxides in human endothelial cells infected by Rickettsia rickettsii. Infect Immun 66: 1293-1298, 1998.

33. Mutunga M, Preston PM and Sumption K: Nitric oxide is produced by Cowdria rumunantium infected bovine pulmonary endothelial cells in vitro and is stimulated by gama interferon. Infect Immun 66: 2115-2121, 1998.

34. Moncada S, Palmer RM and Higgs EA: Nitric oxide: physiology, pathophysiology and pharmacology. Pharmacol Rev 43: 109-142, 1990.

35. Esaki T, Hayashim T, Mutuo E, Kano H, Kumar TN and Asai Y: Expression of iNOS and Fas/Fas ligand correlates with the incidence of apoptotic cell death in atheromatous plaques of human coronary arteries. Nitric Oxide Biol Chem 4: 561-571, 2000.

36. Xu W, Liu L and Charles IG: Microencapsulated iNOSexpressing cells cause tumor suppression in mice. FASEB J 16: 213-216, 2002.

37. Hauser AR and Engel JN: Pseudomonas aeruginosa induces type-III-secretion-mediated apoptosis of macrophages and epithelial cells. Infect Immun 67: 5530-5537, 1999.

38. Bruno TF, Woods DE and Mody $\mathrm{CH}$ : Exoenzyme S from Pseudomonas aeruginosa induces apoptosis in T lymphocytes. $\mathrm{J}$ Leukoc Biol 67: 808-816, 2000.

39. Kaufman MR, Jinghwa J, Zeng L, Ha U, Chow M and Jin S: Pseudomonas aeruginosa mediated apoptosis requires the ADPribosylating activity of ExoS. Microbiol 146: 2531-2541, 2000

40. Rajani S, Cacalano G, Bryan R, Ratner AJ, Sontich CU and van Heerckeren A: Pseudomonas aeruginosa induction of apoptosis in respiratory epithelial cells. Am J Respir Cell Mol Biol 23: 304-312, 2000.

41. Jia J, Alaoui-El-Azher M, Chow M, Chambers TC, Baker H and Jin S: c-Jun NH2-terminal kinase-mediated signaling is essential for Pseudomonas aeruginosa ExoS-induced apoptosis. Infect Immun 71: 3361-3370, 2003.

42. Durieu I, Amsellem C, Paulin C, Chambe MT, Bienvenu J, Bellon B and Pacheco Y: Fas and Fas ligand expression in cystic fibrosis airway epithelium. Thorax 54: 1093-1098, 1999.

43. Yamada T, Goto M, Punj V, Zaborina O, das Gupta TK, Kimbara K and Chakrabarty AM: The bacterial redox protein azurin induces apoptosis in J774 macrophages through complex formation and stabilization of the tumor suppressor protein p53. Infect Immun 70: 7054-7062, 2002.

44. Goto M, Yamada T, Kimbara K, Horner J, Newcomb M, GuptaTK and Chakrabarty AM: Induction of apoptosis in macrophages by Pseudomonas aeruginosa azurin: tumoursuppressor protein p53 and reactive oxygen species, but not redox activity, as critical elements in cytotoxicity. Mol Microbiol 47: 549-559, 2003.

45. Dockrell DH: The multiple roles of Fas ligand in the pathogenesis of infectious diseases. Clin Microbiol Infect 9: 766-779, 2003.

46. Yamaoka-Tojo M, Yamaguchi S, Nitobe J, Abe S, Inoue S and Ozaki N: Dual response to Fas ligation in human endothelial cells: apoptosis and induction of chemokines, IL-8 and monocyte chemoattractant protein-1. Coron Artery Dis 14: 89-94, 2003.

47. Schneider P, Holler N, Bodmer JL, Hahne M, Frei K, Fontana A and Tschopp J: Conversion of membrane-bound Fas (CD95) ligand to its soluble form is associated with downregulation of its proapoptotic activity and loss of liver toxicity. J Exp Med 187: 1205-1213, 1998
48. Suda TH, Hashimoto M, Tanaka T, Ochi T and Nagata S: Membrane Fas ligand kills human peripheral blood $\mathrm{T}$ lymphocytes, and soluble Fas ligand blocks the killing. J Exp Med 186: 2045-2050, 1997

49. Mogi M, Fukuo K, Yang J, Suhara T and Ogihara T: Hypoxia stimulates release of the soluble form of Fas that inhibits endothelial cell apoptosis. Lab Invest 81: 177-184, 2001.

50. Holler N, Tardivel M, Kovacsovics-Bankowski S, Hertig O, Gaide F and Martinon A: Two adjacent trimeric Fas ligands are required for Fas signaling and formation of a deathinducing signaling complex. Mol Cell Biol 23: 1428-1440, 2003.

51. Blaise GA, Gauvin D, Gangal M and Authier S: Nitric oxide, cell signaling and cell death. Toxicology 208: 177-192, 2005.

52. Aktan F: iNOS-mediated nitric oxide production and its regulation. Life Sci 75: 639-653, 2004.

53. Huang X and Hazlett LD: Analysis of Pseudomonas aeruginosa corneal infection using an oligonucleotide microarray. Invest Ophthalmol Vis Sci 44: 3409-3416, 2003.

54. Cherayil BJ, McCormick BA and Bosley J: Salmonella enterica serovar typhimurium-dependent regulation of inducible nitric oxide synthase expression in macrophages by invasins SipB, SipC, and SipD and effector SopE2. Infect Immun 68: 5567-5574, 2000 .

55. Zharikov SI, Sigova AA, Chen S, Bubb MR and Block ER: Cytoskeletal regulation of the L-arginine NO pathway in pulmonary artery endothelial cells. Am J Physiol Lung Cell Mol Physiol 280: L465-L473, 2001.

56. Su Y, Zharikov SI and Block ER: Microtubule-active agents modify nitric oxide production in pulmonary artery endothelial cells. Am J Physiol Lung Cell Mol Physiol 282: L1183-L1189, 2002.

57. Zeng $\mathrm{C}$ and Morrison AR: Disruption of the actin cytoskeleton regulates cytokine-induced iNOS expression. Am J Physiol Cell Physiol 281: C932-C940, 2001.

58. Hattori Y and Kasai K: Disruption of the actin cytoskeleton upregulates iNOS expression in vascular smooth muscle cells. J Cardiovasc Pharmacol 43: 209-213, 2004.

59. Frithz-Lindsten E, Du Y, Rosqvist R and Forsberg A: Intracellular targeting of exoenzyme S of Pseudomonas aeruginosa via type III-dependent translocation induces phagocytosis resistance, cytotoxicity and disruption of actin microfilaments. Mol Microbiol 25: 1125-1139, 1997.

60. Pederson KJ, Vallis AJ, Aktories K, Frank DW and Barbieri JT: The amino-terminal domain of Pseudomonas aeruginosa ExoS disrupts actin filaments via small-molecular-weight GTPbinding proteins. Mol Microbiol 32: 393-401, 1999.

61. Krall R, Schmidt G, Aktories K and Barbieri JT: Pseudomonas aeruginosa ExoT is a Rho GTPasee-activating protein. Infect Immun 68: 6066-6068, 2000.

62. Chang B, Nishikawa M, Sato E and Inoue M: Mice lacking inducible nitric oxide synthase show strong resistance to antiFas antibody-induces fulminant hepatitis. Arch Biochem Biphys 411: 63-72, 2003

63. Niinobu T, Fukuo K, Yasuda O, Tsubakimoto M, Mogi M and Nishimaki H: Negative feedback regulation of activated macrophages via Fas-mediated apoptosis. Am J Physiol Cell Physiol 279: C504-C509, 2000.

64. Fukuo K, Hata S, Suhara T, Nakahashi T, Shinto Y and Tsujimoto Y: Nitric oxide induces upregulation of Fas and apoptosis in vascular smooth muscle. Hypertension 27: 823-831, 1996. 